\title{
Rare Cause of Sepsis - Chromobacterium violaceum - A Case Report
}

\section{B. Subitha ${ }^{1 *}$, T. Jeyamurugan ${ }^{2}$, S. Nithya Gomatheswari ${ }^{1}$ and G. Hariprasad ${ }^{3}$}

Department of Microbiology, Government Thoothukudi Medical College, Thoothukudi, India

\author{
*Corresponding author
}

\section{A B S T R A C T}

Keywords

Sepsis,

Chromobacterium violaceum.

Pencillins and Cephalosporins

Article Info

Accepted:

17 April 2017

Available Online:

10 May 2017
Chromobacterium violaceum is a rare pathogen that causes potentially fatal infections in human beings. It is a normal inhabitant of soil and stagnant water of the tropical and subtropical areas. Chromobacterium violaceum infection usually occurs after broken skin exposed to contaminated soil or water. The organism is mostly resistant to pencillins and cephalosporins and sensitive to fluoroquinolones and aminoglycosides. Rapid progression to sepsis with metastatic abscesses is the striking feature of $C$. violaceum infection .High mortality rates associated with these infections necessitates prompt diagnosis and appropriate antimicrobial therapy. Here the present study reports a case of $C$. violaceum septicemia in a $4 \frac{1}{2}$ years old male child.

\section{Introduction}

Chromobacterium violaceum is a gram negative, motile, facultatively anaerobic, oxidase and catalase positive bacillus that is temperature sensitive and widely distributed in natural aquatic environments (Rajiv Karthik et al., 2012; Lee et al., 1999). Although first identified in 1881, its pathogenic potential was illustrated only in 1905 by Woolley from a fatal case of a buffalo in Philippines. Human case of infection caused by this pathogen was first established by Lessler from Malaysia in 1927 (Pallab Ray et al., 2004; Christopher et al., 2001). It grows easily on ordinary media like blood agar, MacConkey agar, and nutrient agar (Shamshul Ansari et al., 2015). Both pigmented and non-pigmented strains exist, though the non-pigmented strains are rare.
The pigmented strains produce a violet nondiffusible pigment known as violacein, which is soluble in ethanol and insoluble in water and chloroform (Harapriya Kar et al., 2013; Julio Alexander et al., 2007). More than 150 cases have been reported worldwide from Vietnam, Taiwan, Japan, United States, Brazil, Argentina, Australia, Senegal, SriLanka and India (Rajiv Karthik et al.,2012; Pallab Ray et al., 2004;). In most of the cases, the predominant portal of entry appears to be broken skin exposed to the organism through contaminated soil and water (Shamshul Ansari et al., 2015; James Campbell et al., 2013). Main clinical features include sepsis, multiple liver abscesses and diffuse pustular dermatitis, and the possibility of relapse (Kaufman et al., 1986). The microorganism, 
previously thought to be confined to the geographic area between latitudes $35^{\circ} \mathrm{N}$ and $35^{\circ} \mathrm{S}$, may be expanding its habitat beyond this range due to the effects of global warming (Ching-Huei Yang et al., 2011).

\section{Case report}

A $4 \frac{1}{2}$ years old male child was referred to our hospital with complaints of 5 days duration of high grade fever, abdominal pain and respiratory distress. On examination, patient was febrile, and toxic. Examination of respiratory system revealed tachypnoea and bilateral crepitations. Per abdominal examination showed tenderness and mild hepatomegaly and CNS was within normal limits. Blood examination showed $9.9 \mathrm{~g} / \mathrm{dl}$ haemoglobin, total leucocyte count of 2700/mm3 with 75 per cent polymorphs and platelet count $83000 / \mathrm{mm} 3$. Biochemical parameters revealed normal renal functions and elevated liver enzymes. Serological tests for Hepatitis B, C, Dengue, Leptospira, Scrub typhus and HIV were negative. Peripheral smear study was negative for malaria. Chest X-ray showed bilateral infiltrates. Ultrasonography of abdomen confirmed hepatomegaly. A blood culture was sent at admission in brain heart infusion broth which was incubated at $37^{\circ} \mathrm{C}$. Subcultures were done on blood agar, on MacConkey agar, and on nutrient agar. Culture plates were incubated at $37^{\circ} \mathrm{C}$ aerobically. After 24 hours, fine, violet colonies were observed. The organism was a motile, Gram negative rod. It was catalase and oxidase positive and reduced nitrate. It fermented glucose and trehalose but did not ferment lactose or mannitol. Triple sugar iron medium showed an alkaline slant and acid butt without gas and $\mathrm{H} 2 \mathrm{~S}$ production. Citrate was not utilized. Arginine was decarboxylated but not lysine or ornithine. The organism was identified as Chromobacterium violaceum based on biochemical characteristics and pigment production. Antibiotic susceptibility of the organism was tested by disk diffusion method. The results were interpreted as per the Clinical and Laboratory Standards Institute (CLSI) guidelines for other non-Enterobacteriaceae. The isolate was resistant to Ampicillin, Cefotoxime, Ceftriaxone and Imepenam. The organism was sensitive to Ciprofloxacin and Amikacin. The patient progressed to severe respiratory distress, hypotension then shock and finally expired within $48 \mathrm{hrs}$ of admission before the results of antibiotic sensitivity test came through.

\section{Results and Discussion}

Human infections caused by $C$. violaceum are rare. Although C. violaceum is considered nonpathogenic, this bacterium causes systemic infection in previously healthy individuals. Most patients have no underlying disease, but Macher and Mamlok et al., (1997) have found $C$. violaceum to be more common in patients with Chronic Granulomatous Disease and polymorphonuclear leukocyte glucose 6 phosphate dehydrogenase deficiency. Our patients had neither of those conditions.

The dominant route of infection for this pathogen is through exposure of injured skin to contaminated water or soil, with effects ranging from cutaneous lesions and visceral abscesses to severe sepsis, which progresses rapidly to death (Shamshul Ansari et al., 2015 Pallab Ray et al., 2004). Leucopenia as severe as detected in the present patient has been reported in pediatric cases. This is probably due the consequences of the lipopolysaccharide and other toxins produced by the large number of bacilli infecting the tissues. Virulent strains have elevated levels of superoxide dismutase and catalase that may protect the microorganism from phagocytic attack in humans, possibly leading to its extreme virulence (Ching-Huei Yang et al., 2011). 
Fig.1 Violet color pigmented colonies of Chromobacterium violaceum in Nutrient agar

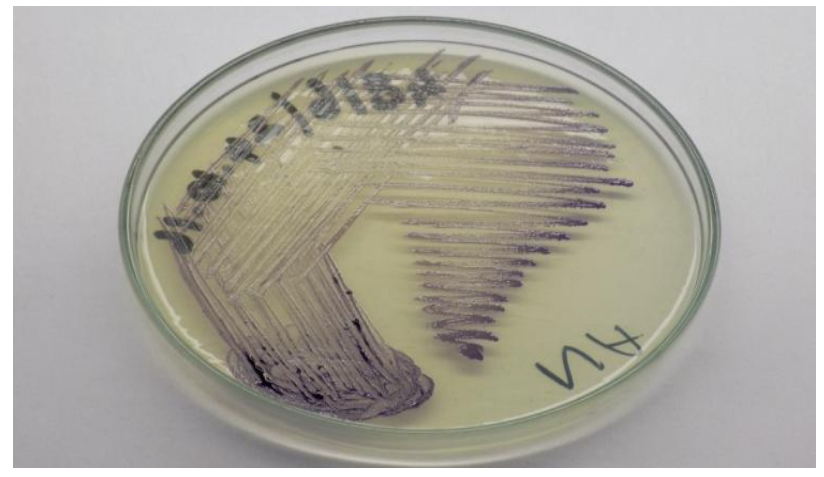

Fig.2 Antibiotic sensitivity pattern of Chromobacterium violaceum

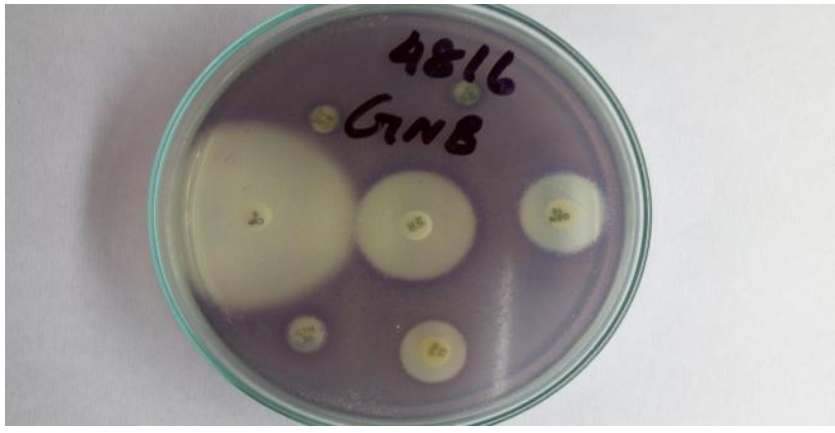

Diagnosing C. violaceum infection is currently based on a culture of clinical specimens followed by subsequent biochemical identification. There is no serological test available for diagnosis. In 2006, Scholz and colleagues developed a method for detecting $C$. violaceum by multiplex polymerase chain reaction, but it has not been widely accepted to date (Vishnu Kaniyarakkal et al., 2016). Data on antimicrobial susceptibility patterns of $C$. violaceum is very limited owing to the rarity of isolation from clinical specimens (Narayan Prasad et al., 2016). Most strains show resistance to penicillins and other beta-lactam antibiotics. According to study by Aldridge, Ciprofloxacin is the most effective antibiotic in vitro (Aldridge et al., 1988). Therefore, it is important for physicians to be aware of $C$. violaceum infection and its appropriate antimicrobial treatment regimen.

In conclusion, though human infections caused by $C$. violaceum are rare, the increasing incidence suggests this to be an emerging pathogen. Thus, from this case study we recommend rapid diagnosis and prompt treatment of infections caused by Chromobacterium violaceum.

\section{References}

Aldridge, K.E., Valainis, G.T., Sanders, C.V. 1988. Comparison of the in vitro activity of ciprofloxacin and 24 other antimicrobial agents against clinical strains of Chromobacterium violaceum. Diagn. Microbiol. Infect. Dis., 10(1): 3139.

Ching-Huei Yang, Yi-Hwei Li. 2011. Chromobacterium violaceum infection: A clinical review of an important but neglected infection, J. Chinese Med. Association, vol 74 435-441.

Christopher, C., Moore Joshua, E., Lane, Jeffrey, L., Stephens. 2001. Successful Treatment of an Infant with 
Chromobacterium violaceum Sepsis. Clin. Infect. Dis., 32(6): e107-e110.

Clinical and Laboratory Standards Institute. Performance Standards for Antimicrobial Disk Susceptibility Tests. 11th Ed. Wayne, PA: Clinical and Laboratory Standards Institute; 2012. pp. M02-A11. Approved Standard.

Harapriya Kar, Vijay Mane, A.D. Urhekar, Samir Pachpute, Anahita Hodiwala. 2013. A first case report in tertiary care hospital, Navi Mumbai, India Chromobacterium violaceum septicaemia in a child. Int. J. Curr. Microbiol. App. Sci., 2(7): 245-249.

James, I., Campbell, Nguyen Phu Huong Lan, Phan Tu Qui, Le Thi Dung, Jeremy J Farrar and Stephen Baker. A successful antimicrobial regime for Chromobacterium violaceum induced bacteremia BMC Infect Dis., DOI: 10.1186/1471-2334-13-4.

Julio Alexander Díaz Pérez, Jorge García, Laura Andrea Rodriguez Villamizar. 2007. Sepsis by Chromobacterium violaceum: first case report from Colombia. Braz. J. Infect. Dis., vol.11 no.4.

Kaufman, S.C., Ceraso, D., Schugurensky, A. 1986. First Case Report from Argentina of Fatal Septicemia Caused by Chromobacterium violaceum. J. Clin. Microbiol., 23(5): 956-958.

Lee, J., J.S. Kim, C.H. Nahm, J.W. Choi, J. Kim, S.H. Pai, K.H. Moon, K. Lee, and Y. Chong. 1999. Two Cases of Chromobacterium violaceum Infection after Injury in a Subtropical Region. $J$. Clin. Microbiol., 37(6): 2068-2070.

Macher, A.M., B.T. Casale and Fauci, A.S. Chronic granulomatous disease of childhood and Chromobacterium violaceum infection in the south eastern United States. Ann. Intern. Med., 97: 5155.

Narayan Prasad Parajuli, Anjeela Bhetwal, Sumitra Ghimire, Anjila Maharjan, Shreena Shakya, Deepa Satyal, Roshan Pandit, and Puspa Raj Khanal. 2016. Bacteremia caused by a rare pathogen Chromobacterium violaceum: a case report from Nepal, Int. J. Gen. Med., 9: 441-446.

Pallab Ray, Jyoti, Sharma, Rungmei, S.K. Marak,, S. Singhi, Neelam Taneja, Raj Kumar Garg \& Meera Sharma. 2004. Chromobacterium violaceum septicaemia from north India. Indian J. Med. Res., pp 523-526.

Rajiv Karthik, Padmaja Pancharatnam, Veeraraghava Balaji. 2012. Fatal Chromobacterium violaceum septicemia in a South Indian adult. J. Infect. Dev. Ctries., 6(10): 751-755.

Shamshul Ansari, Pramod Paudel, Kishor Gautam, Sony Shrestha, Sangita Thapa, and Rajendra Gautam. 2015. Chromobacterium violaceum Isolated from a Wound Sepsis: A Case Study from Nepal Case Rep. Infect Dis., 181946.

Vishnu Kaniyarakkal, Shabana Orvankundil, Saradadevi Karunakaran Lalitha, Raji hazhethekandi, and . 2016. Chromobacterium violaceum Septicaemia and Urinary Tract Infection: Case Reports from a Tertiary Care Hospital in South India, Case Reports in Infect. Dis., Volume article ID 6795743, 4 pages.

\section{How to cite this article:}

Subitha, B., T. Jeyamurugan, S. Nithya Gomatheswari and Hariprasad, G. 2017. Rare Cause of Sepsis - Chromobacterium violaceum A Case Report. Int.J.Curr.Microbiol.App.Sci. 6(5): 17721775. doi: https://doi.org/10.20546/ijcmas.2017.605.192 